\title{
Anatomy of Lumbar Plexus and Implications to Regional Anaesthesiologist
}

\author{
G. Amudha ${ }^{1}$, Sandeep Diwan ${ }^{2}$ \\ ${ }^{1}$ Department of Anatomy, PSG Institute of Medical Sciences and Research, Coimbatore, Tamil Nadu, India. \\ ${ }^{2}$ Department of Anaesthesia, Sancheti Hospital Pune, Maharashtra, India.
}

\section{Abstract}

Lumbar plexus is one of the two nerve plexuses which supply the lower limb. It is formed in the posterior abdominal wall within the psoas major muscle. The branches of the plexus exit via the medial and lateral borders as well as its ventral surface. It is a complex plexus which gives a branch to complete the formation of lumbo sacral plexus. The branches mainly supply the groin, anterior and medial compartments of thigh. They also supply the hip and knee joints. The cutaneous innervation by the branches of lumbar plexus is limited to the anterior, lateral and medial parts of the thigh, medial side of the leg and foot and also the lower part of anterior abdominal wall and perineum. Regional anaesthesia is a highly skilled and precise technique used widely in the patients to reduce the drug usage and decrease the intra and post operative complications. Lumbar plexus block can be used in surgeries related to hip joint and anterior part of thigh and groin. To execute the procedure successfully, sound knowledge in anatomy of lumbar plexus is required.

Keywords: Lumbar plexus, Branches, Regional anaesthesia.

\section{Introduction:}

Lumbar plexus is one of the nerve plexuses which supply the lower limb. It is a plexus which is formed inside the substance of the psoas major muscle and its branches emerge out of the muscle through its medial and lateral borders and also through its anterior surface. It is predominantly formed by the ventral rami of spinal nerves L 1, 2, 3 and 4 . There is a small contribution from the T12 spinal nerve too. [1] The branches of lumbar plexus contribute to the innervation of lower limb predominantly the hip joint, knee joint, anterior and medial compartments of thigh. They also give cutaneous innervation to the anterior, lateral and medial surfaces of thigh, medial surface of the leg and foot. A small area parallel to inguinal ligament in the anterolateral part of lower abdomen and groin also is supplied by its branches. It gives motor innervation to the lower part of muscles of anterior abdominal wall and cremaster. Communication between the sensory branches of lumbar plexus will result in sensory overlap. [2]

\section{Formation:}

The roots of spinal nerves from L1 to L5 emerge in front of the transverse processes of lumbar vertebra. These roots enter into the part of psoas major muscle which is in between the muscle fibres taking origin from the bodies of lumbar vertebra and intervertebral discs and muscle fibres arising from the transverse processes of lumbar vertebra. [4] When traced from body of $\mathrm{L} 1$ to lower lumbar vertebra, the plexus shares the same coronary plane at $\mathrm{L} 1$ but when it comes down, it is more anterior in position. The first four lumbar ventral rami with a contribution from subcostal nerve form the lumbar plexus. The plexus is complex and prone to have variations. The incidence of variations in lumbar plexus is around $20 \%$.

The $\mathrm{L} 1$ ventral rami with a branch from ventral rami of subcostal nerve form a common trunk which divides and give rise to iliohypogastric and ilioinguinal nerves. L1 ventral ramus gives a branch which joins with a branch from L2 ventral rami to form genitofemoral nerve.

The ventral rami of $\mathrm{L} 2 \mathrm{~L} 3$ and $\mathrm{L} 4$ spinal nerves divide into ventral and dorsal divisions. The ventral divisions of L2, L3

Address of Correspondence: Dr. G.Amudha, Department of Anatomy, PSG Institute of Medical Sciences and Research, Coimbatore, India E-mail: ammuramesh@gmail.com

Submitted: May 2021; Reviewed: May 2021; Accepted: June 2021; Published: July 2021

DOI: 10.13107/ijra.2021.v02i02.036 | www.ijrajournal.com |

This is an Open Access article distributed under the terms of the Creative Commons Attribution Non-Commercial-Share Alike 4.0 License

(http://creativecommons.org/licenses/by-nc-sa/4.0) which allows others to remix, tweak, and build upon the work non-commercially as long as appropriate credit is given and the new creation are licensed under the identical terms. 


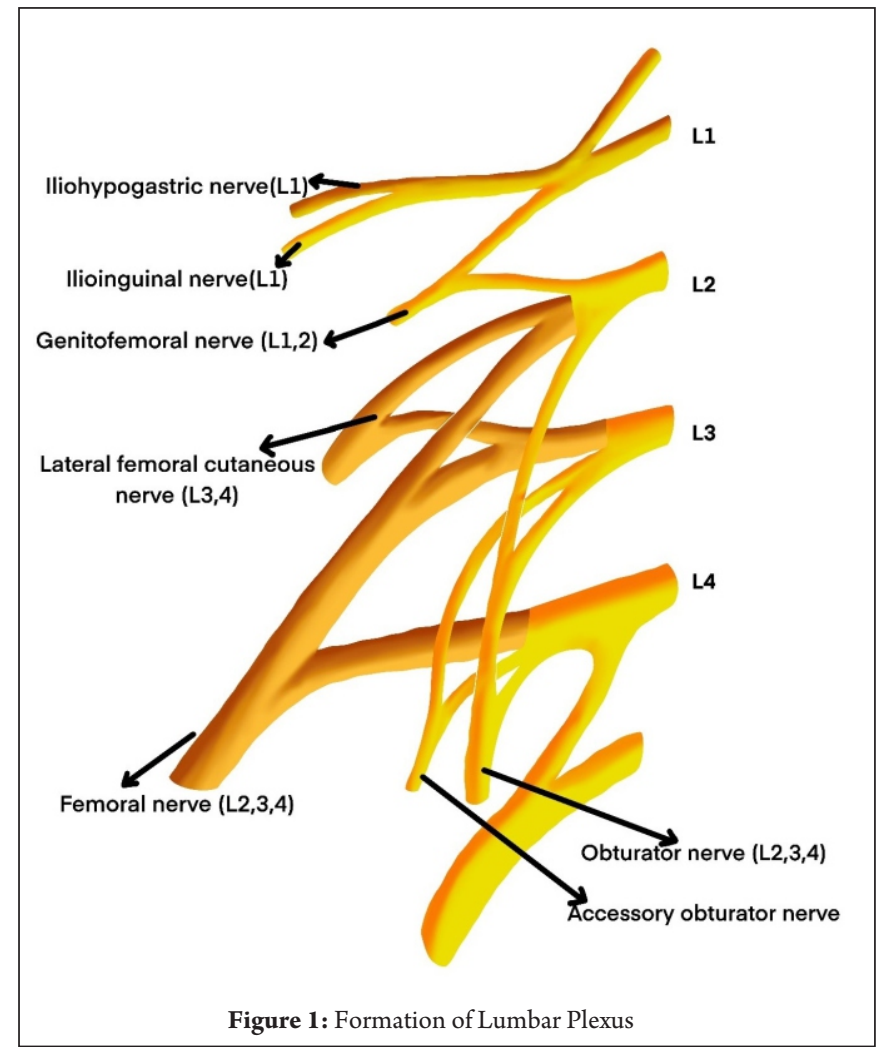

and L4 unite to form Obturator nerve. In 29\% of individuals, L3 and L4 ventral divisions form accessory obturator nerve. The dorsal divisions of L2 and L3 spinal nerves join to form lateral cutaneous nerve of thigh. Dorsal divisions of L2, L3 and $\mathrm{L} 4$ form the femoral nerve.

The L4 ventral rami or the furcal nerve contributes to both lumbar and sacral plexus. After giving a branch to lumbar plexus, it joins with the ventral rami of L5 and forms the lumbo sacral trunk which joins the sacral plexus. $[1,4]$ (Figure 1)

\section{Exit of branches from psoas major:}

There are six constant branches formed from the lumbar plexus. The iliohypogastric and ilioinguinal nerves pass through the lateral border. The lateral cutaneous nerve of thigh and femoral nerve cross the later border in the lower part of psoas major. The genitofemoral nerve pierces the anterior surface and runs over the psoas major. The obturator nerve exits psoas major muscle through its medial border. The inconstant branch, accessory obturator nerve if present exits through the medial border [4]. (Figure 2)

\section{Course and distribution of branches of lumbar plexus: Iliohypogastric nerve:}

It arises from L1 but sometimes from $\mathrm{T} 12$ also and emerges from the upper part of lateral border of psoas major. It lies posterior to medial arcuate ligament near its exit. It runs over the anterior surface of the quadratus lumborum where it is

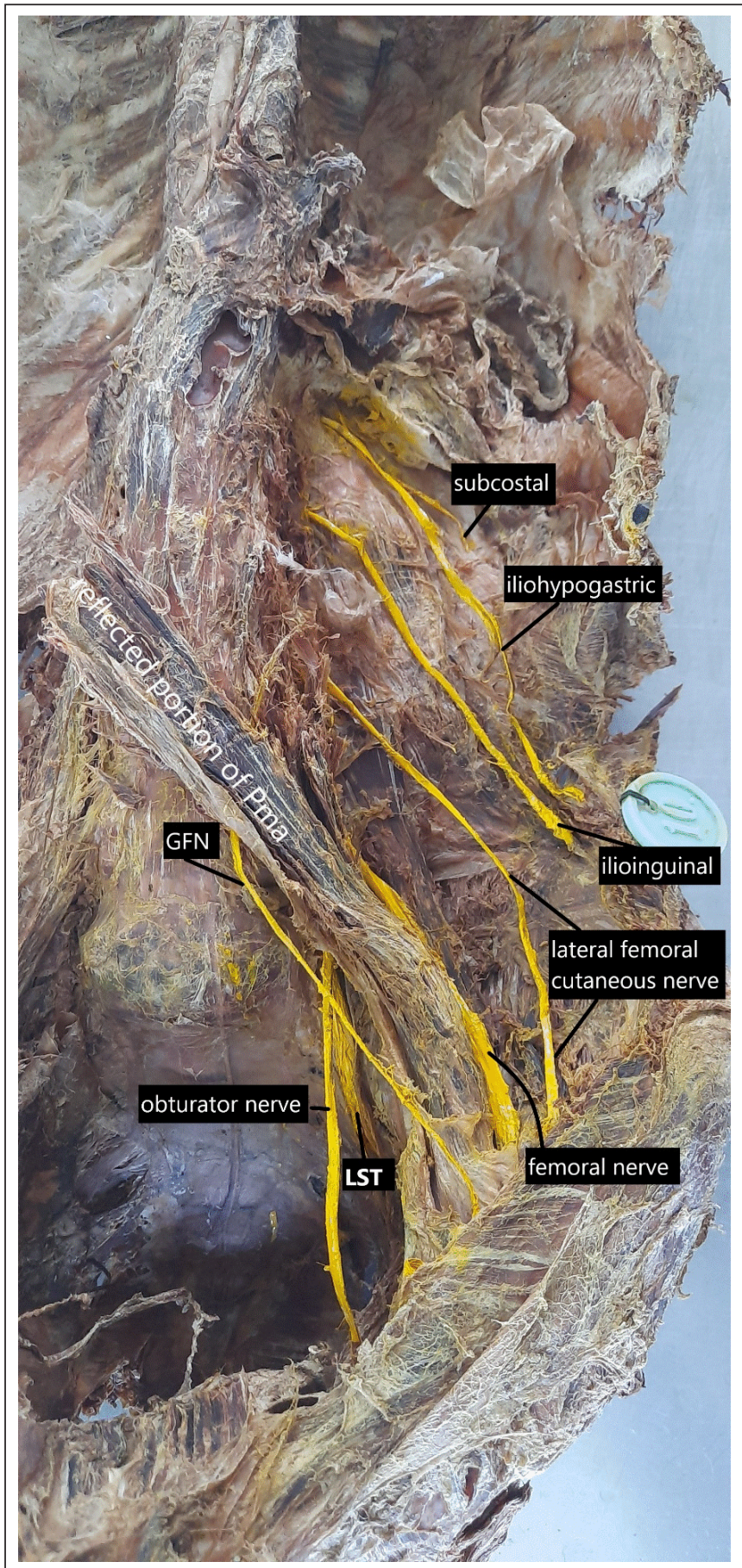

Figure 2: The upper and lower lumbar plexus nerves. The upper lumbar nerves usually are spared during a lumbar plexus block. The lower lumbar plexus nerves in relation to the psoas major is particularly noted for its lateral and medial exit.

related to the lower pole of kidney and reaches the lateral surface of lower part of anterior abdominal wall. Here, it innervates the posterior fibres of transversus abdominis near its origin from iliac crest. After this, it runs anteriorly between transversus abdominis and internal oblique muscles, supplies them. It also gives a lateral cutaneous branch which supplies the posterolateral part of gluteal region. It continues medially and pierces internal oblique 
anteromedial to anterior superior iliac spine, runs in between internal and external oblique muscles. It ends by piercing the external oblique above the superficial inguinal ring and supplies skin over the suprapubic region.

\section{Ilioinguinal nerve:}

It takes its origin from L1 but may get communications from $\mathrm{T} 12$ or L2 and emerges below the iliohypogastric nerve in the lateral border of psoas major. Its courses obliquely over the quadratus lumborum and iliacus. After that it reaches transversus abdominis and pierces it inferomedial to anterior superior iliac spine. It supplies transversus abdominis and internal oblique before it enters the inguinal canal where it travels superficial to its contents. It exits the inguinal canal through superficial inguinal ring and gives cutaneous innervation to the root of the penis and scrotum in males, mons pubis and labia majora in females and upper medial part of the thigh in both sexes.

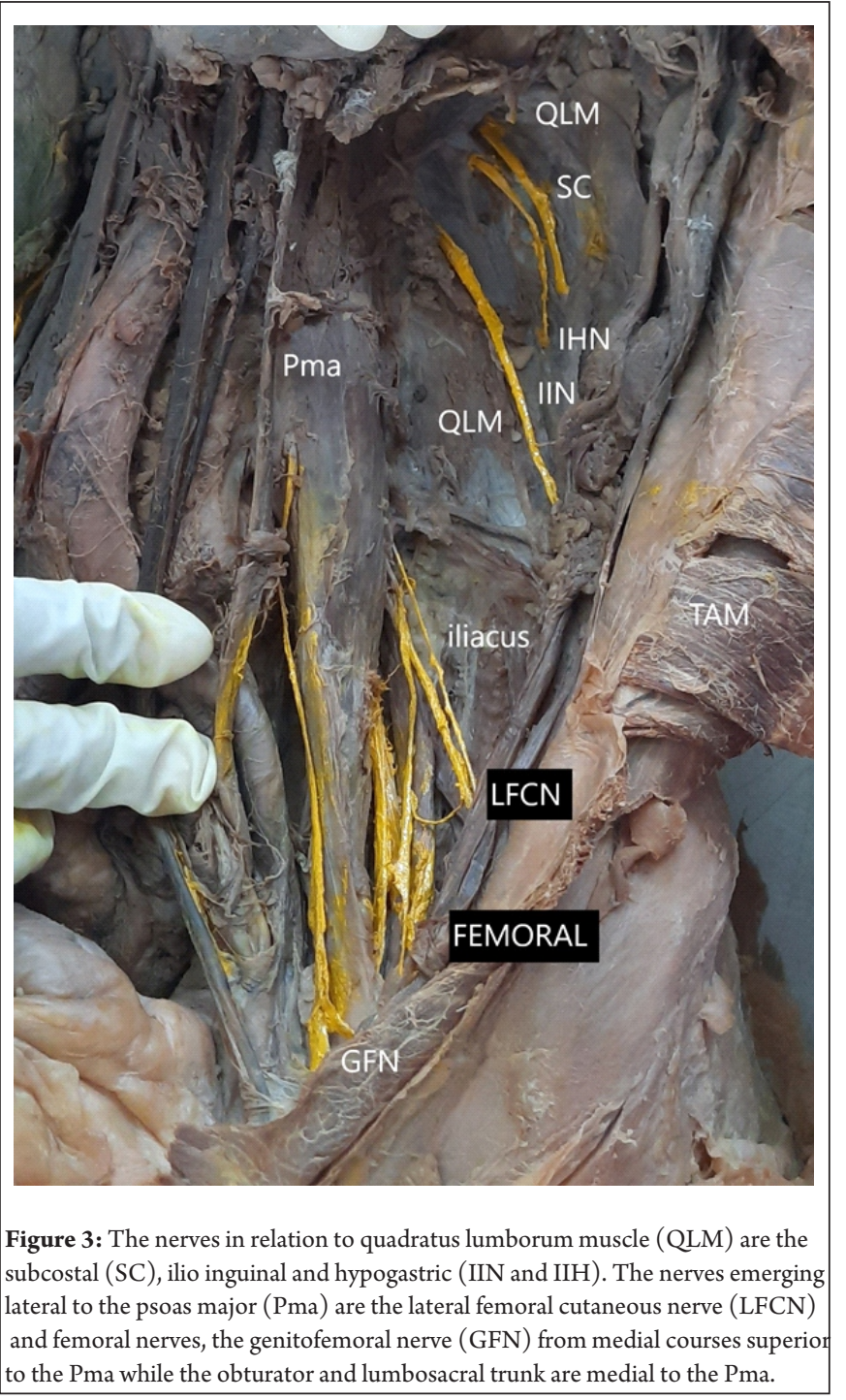

\section{Lateral femoral cutaneous nerve:}

It usually arises from dorsal divisions of L2 and L3. It emerges from the lateral border of psoas major and runs obliquely over the iliacus muscle before it reaches the anterior superior iliac spine. In the iliac fossa the nerve lies posterior to caecum in the right side and descending colon in the left side beneath the parietal peritoneum. It crosses behind inguinal ligament medial to anterior superior iliac spine and enters the thigh anterior to Sartorius. Here in the thigh it divides into anterior and posterior branches. The anterior branch innervates skin over the anterior and lateral part of thigh up to the knee joint and takes part in the formation of peripatellar plexus. The posterior branch pierces the fascia lata and supplies skin over the greater trochanter and adjacent gluteal region.

\section{Femoral nerve:}

It is formed by dorsal divisions of L2, L3 and L4 and emerges from the lateral border of psoas major about $4 \mathrm{~cm}$ above inguinal ligament. It runs in the groove between psoas major and iliacus below the inguinal ligament and reaches the thigh lateral to femoral sheath. Here it divides in to anterior and posterior divisions before supplying the anterior compartment of thigh. It supplies the muscles of anterior compartment of thigh and skin over the anterior surface of thigh and medial side of leg and foot. (Figure 3 )

\section{Genito femoral nerve:}

It is formed by $\mathrm{L} 1$ and $\mathrm{L} 2$ and exits psoas major muscle by piercing its anterior surface opposite third or fourth lumbar vertebra. It runs beneath the parietal peritoneum and crosses the ureter before dividing in to genital and femoral branches. The genital branch crosses external iliac artery and enters the deep inguinal ring. It traverses behind the contents of inguinal canal and exits through the superficial inguinal ring where it ends by supplying cremaster muscle and skin over the external genitalia. The femoral branch runs lateral to external iliac artery and enters the femoral sheath behind the inguinal ligament and lateral to femoral artery. It pierces the femoral sheath and ends by supplying the skin over the femoral triangle.

\section{Obturator Nerve:}

It arises from the ventral divisions of L2, L3 and L4 and emerges from the medial border of psoas major muscle opposite to fifth lumbar vertebra. It runs behind the common iliac vessels and lateral to internal iliac vessels. It crosses the pelvis in its lateral wall where it is anterosuperior to obturator vessels before reaching obturator foramen. After crossing obturator foramen it enters the medial 


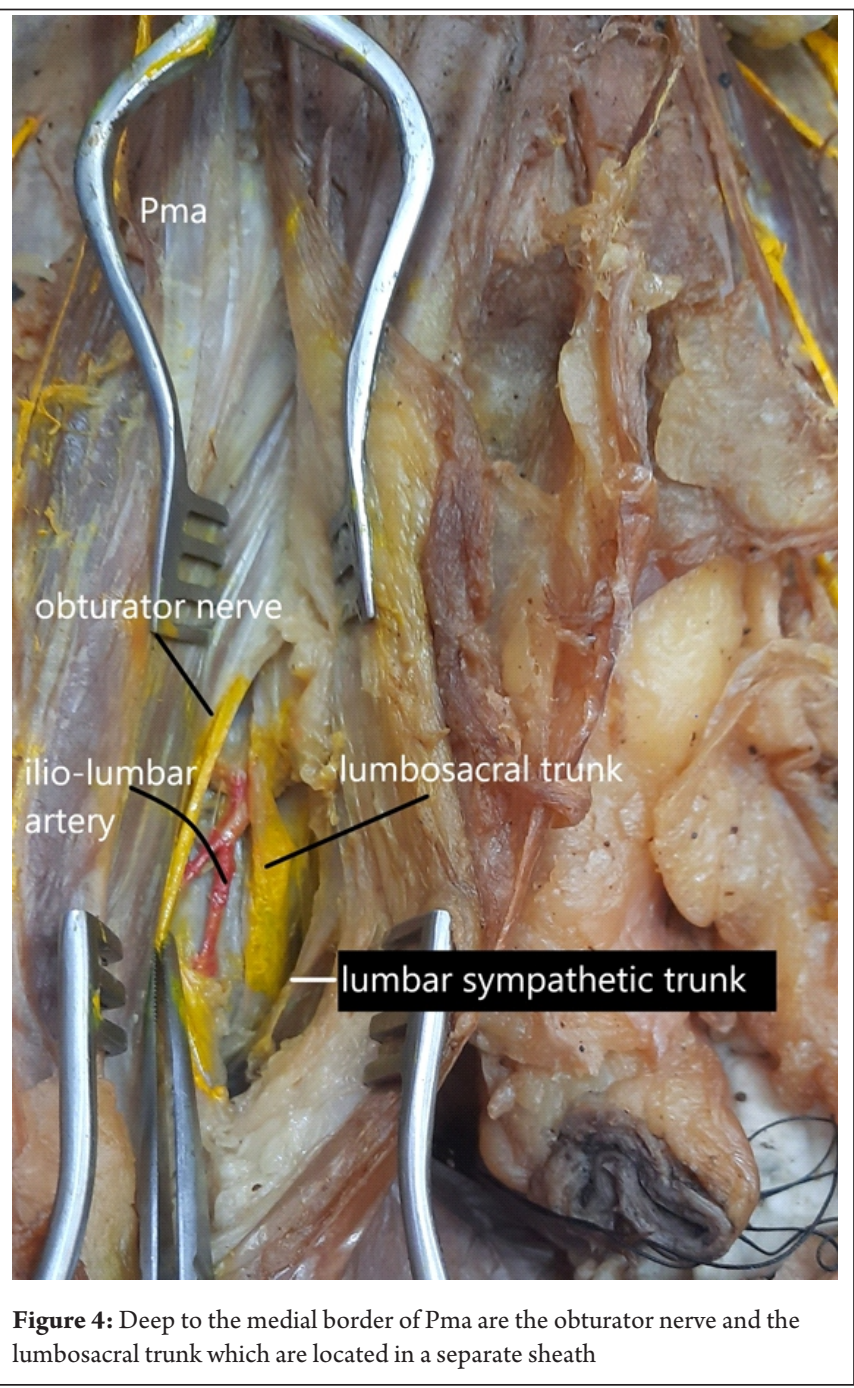

compartment of thigh where it is divided into anterior and posterior divisions. It supplies the muscles of adductor compartment of thigh, hip and knee joints. (Figure 4)

\section{Accessory obturator nerve:}

It is a small and rare branch. It arises from ventral rami of $\mathrm{L} 3$ and L4. It too courses along the medial border of psoas major. When it reaches the superior pubic ramus it runs posterior to pectineus. It branches out here; one of them supplies hip joint, the other supplies pectineus. The third branch joins the anterior division of obturator nerve. But the branching pattern is not constant.

\section{Cross-Section at the level of $\mathrm{L} 3-4$}

A cross-section at L3-4 reveals the location of the lumbar nerve root in the psoas major. As it emerges from the intervertebral foramina the lumbar nerve root lies initially in the lumbar paravertebral space, as it moves laterally and it descends in the posterior one third of the psoas major. Thus, the site of the needle tip during a lumbar plexus block would be the posterior and medial quadrant. Figure 5 Another

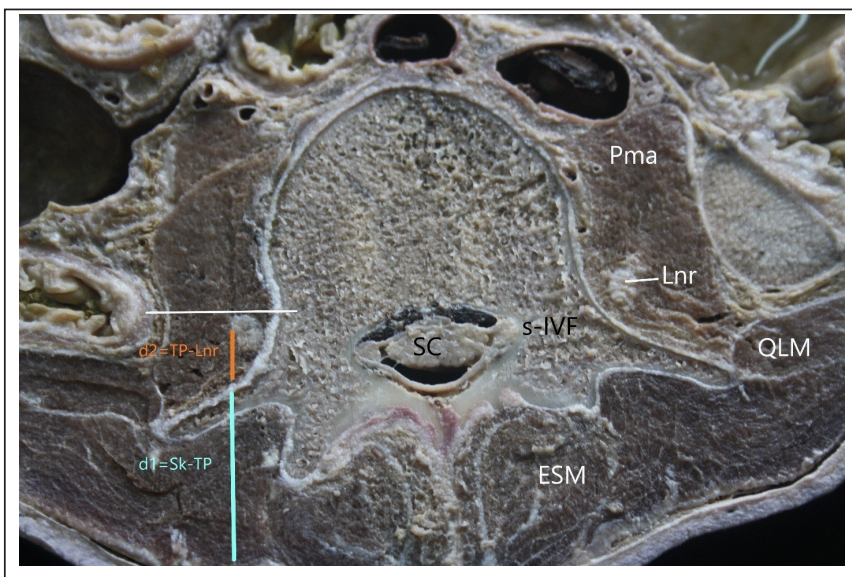

Figure 5: Cross-section at L3-4 depicting the lumbar nerve root (Lnr) in the posterior and medial quadrant of the psoas major (Pma). The skin to transverse process (TP) distance $\mathrm{d} 1$ will be variable but the TP to $\mathrm{LNr}$ distance $\mathrm{D} 2$ will be around $2 \mathrm{~cm}$. QLM-quadratus lumborum muscle; ESM-erector spinae muscle; s-IVF- site for intervertebral foramina; SC-spinal cord ( Cross-sections were performed at department of anatomy, Bharatividyapeeth Deemed University, Pune, Maharashtra, India.)

important point to be noted is the distance from the transverse process to the lumbar plexus is $1.8-2.2 \mathrm{~cm}$, thus, it is obligatory not to exceed a distance of $2 \mathrm{~cm}$ after the transverse process is encountered which itself serves an important anatomical landmark.

\section{Conclusion:}

Though anatomical variations are not uncommon in lumbar plexus, the major six branches and area of their innervation are constant. The anatomical plane inside the psoas major muscle (posterior one third)gives the clinician to use it as a point to administer lumbar plexus block.The transverse process is an important osseous anatomical landmark and the lumbar plexus is situated predictably at a distance of $2 \mathrm{~cm}$ in front of the transverse process in the posterior and medial quadrant of Pma. [8] A thorough anatomical knowledge regarding the formation and distribution of lumbar plexus will be helpful while considering alternative anesthesia techniques in surgeries involving anteromedial parts of thigh hip joint, knee joint and perineum. The lumbar plexus block can also be used for post operative pain management and in conditions like neuropathies and meralgia paresthetica. $[3,9,10]$ 
Declaration of patient consent: The authors certify that they have obtained all appropriate patient consent forms. In the form, the patient has given his consent for his images and other clinical information to be reported in the Journal. The patient understands that his name and initials will not be published, and due efforts will be made to conceal his identity, but anonymity cannot be guaranteed.

Acknowledgment: Dr A.K.Manickavasuki: Associate Professor of Anatomy, PSG Institute of Medical Sciences and Research, Coimbatore who did the dissection and photographed Lumbar plexus in the cadaver.

Dr Purshottam Manvikar: Head of department for the cross-section at the level of L3-4.

Dr Rammurthy Kulkarni: Consultant Anaesthesiologist, Axon Associates, Bengluru for Figure 1.

Conflict of interest: Nil Source of support: None

\section{References}

1. Standring S, Gray's Anatomy The anatomical Basis of Clinical Practice.41st ed. Elsevier; 2015.

2. Mahakkanukrauh $P$ et al. A cadaveric study of the anatomical variations of the lumbar plexus with clinical implications J of Anatomical Society of India, 2016;65:24-28.

3. Javier J. Polania Gutierrez ; Bruce Ben-David .2020. Lumbar plexus block. https://www.ncbi.nlm.nih.gov/books/NBK556116. (Last accesses date: $3^{\text {rd }}$ June 2021)

4. Philip A Anloague, Peter Hujibregts. Anatoical variations of the lumbar plexus: A descriptive anatomy study with proposed clinical implications. The Journal of manual and manipulative therapy 2009; 17:e107-e114.

5. Deepti Arora, Subhash Kaushal, Gurbachan Singh. Variations of lumbar plexus in 30 adult human cadavers - A unilateral prefixed plexus. Int. J of Plant, Animal and Environmental sciences. 2014; 4:225-228.

6. Prof. Gamal S Desouki et al 2016 Study of anatomical pattern of lumbar plexus in human (cadaveric study).Az.J.Pharm Sci. 2016; 54:54-69.
7. Dr. Fasila P. Asis, Dr.Priya Ranganath A Human cadaveric study on variations in formation and branching pattern of lumbar plexus with its clinical implications. Scholars J of App. Med. Sci. 2017;58-63.

8. Xavier Capdevila, Philippe Macaire, Christophe Dadure, Olivier Choquet, Philippe Biboulet, Yves Ryckwaert, Françoise D'Athis Continuous psoas compartment block for postoperative analgesia after total hip arthroplasty: new landmarks, technical guidelines, and clinical evaluation Anesth Analg 2002 Jun;94:1606-13.

9. Ahiskalioglu A, Tulgar S, Celik M, Ozer Z, Alici HA, Aydin ME. Lumbar Erector Spinae Plane Block as a Main Anesthetic Method for Hip Surgery in High Risk Elderly Patients: Initial Experience with a Magnetic Resonance Imaging. Eurasian J Med 2020; 52: 16-20.

10. Chayen D, Nathan H, Chayen M. The psoas compartment block. Anesthesiology. 1976 Jul; 45:95-9.

How to cite this article: Amudha G, Diwan S | Anatomy of Lumbar Plexus and Implications to Regional Anaesthesiologist | International Journal of Regional Anaesthesia | July-December 2021; 2(2): $102-106$. 